\title{
Editorial
}

\section{Why: gene therapy?}

Although science is the systematic study and formulation of knowledge, it shares with the 'arts', a remarkable susceptibility to fashions and trends. These in turn can be manipulated not only by current events (eg epidemics and geological catastrophes) but also by journal editors and grant committees. Any student of medicine (its art or its science) rapidly becomes impressed with the empirical nature of its practice and the incompleteness of our understanding of many chronic diseases. Indeed many conditions are merely managed on a symptomatic basis because of a lack of specific effective treatments. (For any common incurable condition there is no shortage of ineffective, sometimes toxic and often expensive treatments.)

In the last two decades or so, treatment has been developed on a more scientific basis such as studying the basic biologies of the sympathetic and acid secreting symptoms which gave us (courtesy of Sir James Black) beta blockers and the $\mathrm{H}_{2}$ antagonists, respectively.

Even defining the science behind the disease, which in both cases was the discovery of the relevant receptor which when blocked led to the control of disease, the optimal drug to block it was defined by function in an assay screening large libraries of compounds. Only relatively recently has the structure of receptors been elucidated enough to enable computers to design candidate blocking compounds or peptides.

Clinical medicine is now starting to benefit from the enormous investment in the basic (as opposed to applied) sciences which occurred in the early 1970s which at the time appeared to have no obvious therapeutic goal. One of the best candidate therapies poised to reap the harvest that basic science investment has sown is gene therapy. The need for other therapies is obvious to any 'chronic' patient whether they suffer from a genetic disorder such as cystic fibrosis, or whether they suffer from a chronic treatable but not curable condition such as hypertension, ischaemic heart disease, diabetes, asthma, convective tissue disorders (including rheumatoid arthritis), chronic (and progressive) neurologic conditions such as Parkinson's disease, motor neuron disease, multiple sclerosis and the dementias, as well as the cancers that will inevitably afflict over one-third of aging populations.

The pastiche concept of gene therapy is wonderfully simple and compellingly attractive to the frustrated clinicians who have to deal with the aforementioned conditions. The fact that the tools exist to apply some of the more simple theoretical approaches to the clinic has led to overly optimistic projections (hype) as to what can eventually be achieved (as early as next year!). The find- ing that the approach is riddled with 'technical problems', which to the pessimist may seem insurmountable has led to the inevitable backlash (which does not appear to have so far affected funding opportunities), from those who expected too much too soon, for such a young science. However, this approach can easily be overdone with both hilarious and tragic consequences, for example no less a scientist than the president of the Royal Society proclaimed that heavier than air flying machines were a contradiction in terms and would never fly, and the aeronautical establishment's reception of Frank Whittle's design for the jet engine was so overly cynical and hostile it drove its development overseas with all the resultant economic and social ramifications.

Although detailed study of the application of gene therapy to a number of disease conditions throws up more questions than answers, such as how to infect specific and in the case of cancer 'all' relevant cells, how best to administer the gene, how to prevent DNA integration, how to achieve prolonged expression etc; pursuing curiosity driven and emphatical basic research has already given us at least two reasons to be optimistic if not cheerful. The first reason is that in the case of genetic replacement conditions such as the replacement of the ADA gene in the case of severe combined immunodeficiency (SCID) children, only a small percentage of the normal amount of the enzyme need be expressed to 'cure' the phenotype of disease, thus overcoming one of the major predicted impossibilities, ie that of completely replacing all the biologically available ADA in a normal individual. In retrospect this could have been anticipated from our knowledge of hemophilia, as children with less than 5\% of the normal level of factor VIII are to all extents and purposes normal until they present with a swollen (blood-filled) joint following trauma on the sportsfield. The second reason to be hopeful is the dissemination of the bystander effect in the application of virally directed enzyme prodrug therapy (VDEPT) to cancer. Whereas it is obvious that gene replacement for a deficiency will be quite satisfactory even if a $100 \%$ replacement is not achieved, this is not the case with many tumours. Indeed, many of the major solid tumours can be reduced in bulk by an apparent $100 \%$, only to occur again months to years later. The inability of most delivery systems to 'infect' all tumour cells has led to scepticism that such approaches would suffer from the same limitations as chemotherapy and radiotherapy (ie the inability to infect all cells will result in total loss of control of the tumour in vivo). Happily this appears not to be the case; although the mechanism of the bystander effect is far from clear it would appear that at least one component is effected by the 
immune response as tumour containing activity is not seen in nude mice.

It therefore appears that the extremely high hurdles that we have set ourselves for the application of gene therapy to the clinic may not be as high as previously perceived, which will bring hope not only to oncological application but also to cardiovascular and neurovascular, as well as chronic infectious disease gene therapy strategies. These should be cautiously encouraged where current therapeutic inhibition leads to the question why gene therapy being rhetorically answered as gene therapy why not?
If in doubt remember that according to the scientific dogma and relevant expert establishment views of the earlier part of the century, jet airliners were a scientifically justifiable practical impossibility.

AG Dalgleish

Division of Oncology Department of Cellular and Molecular Sciences St George's Hospital Medical School Jenner Wing Cranmer Terrace London SW17 ORE

The Editors welcome contributed editorials on areas of topical interest. Editorials should be about 1200 words in length with a maximum of five references where relevant. Please submit all contributions to either of the Editorial Offices. 